Check for updates

Cite this: RSC Adv., 2017, 7, 42783

Received 27th July 2017

Accepted 21st August 2017

DOI: 10.1039/c7ra08322j

rsc.li/rsc-advances

\section{Combined effect of $\alpha$-nucleating agents and glass fiber reinforcement on a polypropylene composite: a balanced approach $\dagger$}

\author{
Linfang Cui, ${ }^{\text {ab }}$ Penglei Wang, ${ }^{\text {ab }}$ Yagang Zhang, (D) *abc Letao Zhang, ${ }^{\text {ab }}$ Yurong Chen, ${ }^{\text {ab }}$ \\ Lulu Wang, ${ }^{\text {ab }} \mathrm{Li} \mathrm{Liu}^{\mathrm{a}}$ and Xinfeng Guo
}

\begin{abstract}
Herein, we demonstrated the mechanical properties and morphologies of modified polypropylene (PP) using a combination of glass fibers (GFs) and $\alpha$-nucleating agents (TMP- 6 or TMA-3) at different loadings with the aid of a compatibilizing agent during melt compounding and compression molding. The structural characteristics and properties of modified PP composites were analyzed. The results indicated that TMP-6/GF/PP and TMA-3/GF/PP exhibited significantly enhanced mechanical performance as compared to TMP-6/PP, TMA-3/PP, and neat PP. The influence of the type and concentration of $\alpha$-NAs on the morphology and crystallization behavior of PP composites was also studied. Compared with that of the GF/PP composite, the impact strength of TMA-3/GF/PP increased up to $179.4 \mathrm{~J} \mathrm{~m}^{-1}$ at $0.2 \mathrm{wt} \%$ of TMA-3 with moderate improvements in tensile strength and flexural modulus. Scanning electron microscopy (SEM) revealed that there was good adhesion at the interface between the GFs and PP matrix. This method provided an alternative approach for the design of novel PP composites.
\end{abstract}

\section{Introduction}

As one of the most versatile thermoplastic polymeric materials, polypropylene (PP) has been widely used over the last few decades. ${ }^{1,2}$ It has been found that PP can have several basic crystalline forms such as monoclinic $\alpha$-crystal, trigonal $\beta$-crystal, orthorhombic $\gamma$-crystal, and smectic mesophase. ${ }^{3,4}$ It is well known that the properties of PP strongly depend on its crystal forms. ${ }^{5}$ Compared with other crystalline forms, the $\alpha$-crystalline form offers an interlocked cross-hatch structure, allowing for difficult inter-lamellar slippage and thus results in an enhanced stiffness (tensile and flexural properties). ${ }^{6}$ The $\alpha$-crystal can be obtained under the normal processing conditions. However, the limitation of this approach is the low crystallization rate and unsatisfactory mechanical performance for applications, such as in household electric appliances, and automobile accessories, requiring high comprehensive mechanical properties (tensile strength, flexural modulus, and impact strength). This significantly limits its practical application in the field of advanced functional materials.

\footnotetext{
${ }^{a}$ Xinjiang Technical Institute of Physics and Chemistry, Chinese Academy of Sciences, Urumqi 830011, China. E-mail: ygzhang@ms.xjb.ac.cn; Fax: +86-991-3838957; Tel: +86-18129307169

${ }^{b}$ University of Chinese Academy of Sciences, Beijing 100049, China

'Department of Chemical \& Environmental Engineering, Xinjiang Institute of Engineering, Urumqi 830023, China

$\dagger$ Electronic supplementary information (ESI) available. See DOI: $10.1039 / \mathrm{c} 7 \mathrm{ra} 08322 \mathrm{j}$
}

For this reason, a number of efforts have been focused on the modification of PP to improve the crystallization rate and mechanical performance. The addition of $\alpha$-nucleating agents $(\alpha$-NAs) to PP is considered to be one of the most efficient and feasible ways to improve the mechanical properties and crystallization rate. ${ }^{6-8}$ To date, a large number of compounds have been employed as $\alpha$-NAs, and the most widely used $\alpha$-NAs are sorbitol derivatives, organic phosphate salts, and organic carboxylic acid salts. ${ }^{6-11}$ Sorbitol derivatives were mainly used as clarifiers for improving the transparency of PP due to their high efficiency, but only a slight improvement in the mechanical properties was observed..$^{12}$ It was found that phosphate salts and organic carboxylic acid salts could significantly improve the mechanical properties and crystallization rate of PP. ${ }^{8,11}$ The organic phosphate salts were recognized as promising $\alpha$-NAs for PP. Specifically, 2,2'-methylene-bis(4,6-di-tert-butylphenyl) phosphate (NA-11), as the second generation of organic phosphate salts, was commonly used and proven to be effective to improve the strength and modulus of PP. ${ }^{8}$ Recently, bis $\left[2,2^{\prime}\right.$ methylenebis (4,6-di-tert-butylphenyl)phosphate] aluminum hydroxide salt (NA-21) has been reported as a representative $\alpha$-NA of the third-generation $\alpha$-NAs and has shown improved nucleation for PP as compared to the second-generation $\alpha$-NAs. With regard to the organic carboxylic acid salts, Cai et al. ${ }^{11}$ reported an enhancement in comprehensive mechanical and optical properties and increased crystallization temperature via the bicyclo[2.2.1] heptane dicarboxylate (BCHE13)-induced nucleation of PP, and the results indicated that BCHE13 was an effective nucleating agent. Although PP nucleated with $\alpha$-NAs 
showed an enhancement of the tensile and flexural properties, most of the times, reduced toughness was observed because of its intrinsic structure. ${ }^{\mathbf{1 3 , 1 4}}$ In this regard, it would be highly desirable to develop strategies for PP composites with improved tensile strength and flexural modulus without sacrificing its toughness. It is known that via the addition of fibers to PP, the morphology and crystallization behavior at the interface can be adjusted and the performance of the PP composites can be improved. Compared with other fibers, glass fiber (GF)reinforced PP composites exhibited excellent mechanical performance and increased heat distortion temperature (HDT). It has been used in various fields such as in construction, marine, automotive, and so on. ${ }^{15}$ Interestingly, it was reported that via addition of glass fibers to the PP matrix, not only the tensile strength and flexural modulus, but also the toughness was improved to some extent. ${ }^{\mathbf{1 6 - 1 9}}$ For example, Patcharaphun et $a l .{ }^{17}$ reported that the toughness and stiffness of PP could be enhanced with the reinforcement of GFs. In addition, it was known that the mechanical properties of composites were closely related to the interface adhesion. ${ }^{20}$ The importance of the interface/interphase properties of a polymer matrix was highlighted and discussed in detail by Kim et $a .^{21}$ and Jesson et al. ${ }^{22}$ However, owing to the non-polar nature of PP and polar functional groups on GFs, the interfacial adhesion between the GFs and PP matrix is usually weak. Several methods including surface treatments of GFs, ${ }^{23}$ oxygen plasma treatments of $\mathrm{PP},{ }^{24}$ and the introduction of interfacial compatibilizers to the PP matrix $^{16,25-27}$ have been developed to improve the interfacial adhesion between the GFs and the polymer matrix; among these, the introduction of interfacial compatibilizers has been considered to be an effective method in this regard. Maleic anhydride-grafted polypropylene (PP-g-MA) was developed as an interfacial compatibilizer. ${ }^{28}$ Mohanty et al. ${ }^{29}$ demonstrated that PP-g-MA showed a positive effect on the interfacial properties of the GF-reinforced PP composites. This was attributed to the improved compatibility between the PP matrix and the compatibilizer chains as well as the hydrogen bonding and covalent bonding between GFs and maleic anhydride. Theoretically, the addition of the individual $\alpha$-NAs and GFs can improve the performance of PP; thus, it would be very interesting to find out whether there is a synergistic effect of $\alpha$-NAs and GFs on the mechanical properties and crystallization behavior of PP. However, to the best of our knowledge, study on the combined effects of $\alpha$-NAs and GFs on the performance of PP has been rarely reported.

In this regard, herein, PP nucleated with the $\alpha$-NAs TMP-6 and TMA-3, PP modified by glass fibers (GF/PP), and PP composites modified simultaneously by $\alpha$-NAs and GF (TMP-6/ GF/PP and TMA-3/GF/PP) were designed and prepared via a twostep process. The role of GFs and $\alpha$-NAs in TMP-6/GF/PP and TMA-3/GF/PP and the effects of GFs and $\alpha$-NAs on the morphology, structure, crystallization behavior, and the mechanical performance of PP were investigated in detail by scanning electron microscopy (SEM), X-ray diffraction (XRD), differential scanning calorimetry (DSC), heat distortion temperature (HDT), and polarized optical microscopy (POM). Finally, the influence of the type and concentration of $\alpha$-NAs on the crystallization behavior and mechanical performance, including tensile, flexural, and impact properties, of PP composites was also studied and discussed.

\section{Experimental}

\subsection{Materials}

All materials and chemicals were commercially purchased and used as received unless otherwise notified. The isotactic polypropylene (trade mark K8003) was purchased from CNPC Dushanzi Petrochemical Co. Ltd., China, with a density of $0.9 \mathrm{~g} \mathrm{~cm}^{-3}, M_{\mathrm{w}}=4.4 \times 10^{5} \mathrm{~g} \mathrm{~mol}^{-1}, M_{\mathrm{w}} / M_{\mathrm{n}}=4.1$, and melt flow rate (MFR) of $2.5 \mathrm{~g} \mathrm{~mol}^{-1}$. Antioxidant Irganox 1010 and Irganox 168 were obtained from Ciba Specialty Chemicals (Shanghai, China). The E-glass fibers (with an average diameter of $13 \mu \mathrm{m}$ and a length of $4.5 \mathrm{~mm}$ ) were kindly supplied by Jushi Group (Tongxiang, Zhejiang, China). Maleic anhydride-grafted PP (PP-g-MA, 0.8-1.0 wt\%) was obtained from Nantong Sunny Polymer New Material Technology Co. Ltd., China, and used as a compatibilizer. The commercially available $\alpha$-nucleating agents with the brand name TMP-6 (Fig. 1a) and TMA-3 (Fig. 1b) were purchased from Shanxi Provincial Institute of Chemical Industry, China. Furthermore, the morphologies of TMP- 6 and TMA-3 were characterized by SEM, as shown in Fig. 1a and b. TMP-6 showed a flake shape, and TMA-3 showed an aggregated rod-like shape.

\subsection{Sample preparation and test specimens}

The test specimens were prepared via two-step processes: compounding and compression molding. Before compounding, all materials were completely dried at $60{ }^{\circ} \mathrm{C}$ for $24 \mathrm{~h}$. The melt compounding process was conducted using a co-rotating twin screw extruder (SHJ-20, Nanjing Giant Machinery Co., Ltd., Nanjing, China) at $160-220{ }^{\circ} \mathrm{C}$ from the hopper to the nozzle. The screw diameter of the extruder is $20 \mathrm{~mm}$ and its length-to-diameter $(L / D)$ ratio is 40 . First, a master batch containing PP pellets with different concentrations of $\alpha$-nucleating agents $(0.1,0.2$, and $0.3 \mathrm{wt} \%)$ was compounded through blending in a twin screw extruder at a screw speed of $150 \mathrm{rpm}$. The extruded strands were cooled in water and pelletized with a strand cutter. The obtained pellets were dried at $80^{\circ} \mathrm{C}$ for $12 \mathrm{~h}$. Second, the master batch was melt blended with the glass fibers (20 wt\%) using the same process, but the rotational speed was reduced to $100 \mathrm{rpm}$ to minimize fiber damage. The

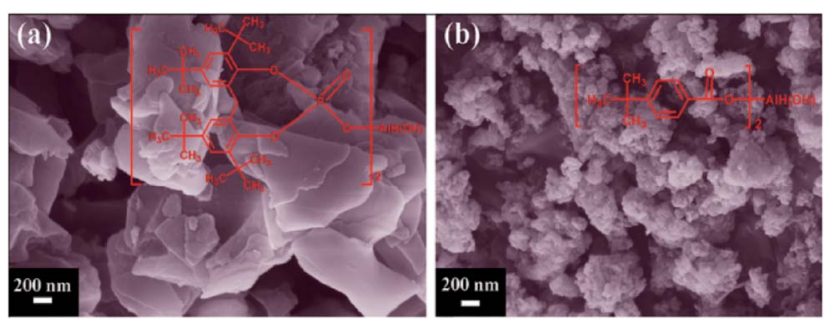

Fig. 1 SEM images of (a) TMP- 6 and (b) TMA-3, with the chemical structures of TMP- 6 and TMA-3 shown on their respective image. 
compositions of the corresponding binary and ternary blends are summarized in Table 1 . To stabilize the resin during melt processing, 0.2 wt\% antioxidant Irganox 1010/Irganox 168 at a ratio of $1 / 1(\mathrm{w} / \mathrm{w})$ was added to $\mathrm{PP}$. The dried pellets were compress-molded using a hot-press (R-3212, Wuhan Qien Science \& Technology Development Co., Ltd, China) at a molten temperature of $220{ }^{\circ} \mathrm{C}$ for $10 \mathrm{~min}$ without the application of to ensure complete melting of the pellets. After this, the materials were pressed under a pressure of $40 \mathrm{MPa}$, and hold temperature and hold time were set at $220{ }^{\circ} \mathrm{C}$ and $30 \mathrm{~min}$, respectively. Finally, the sample was cooled down to room temperature under the cooling circulation system of cold water. All the materials were subjected to the same processes to maintain a consistent processing condition. For comparative purposes, neat PP and binary GF/PP composites were also prepared under the same conditions to ensure sample consistency.

\subsection{Characterization of the PP composites}

2.3.1 Mechanical testing. Standard tensile measurements and flexural tests were conducted using a universal testing machine (UTM, C43-104, MTS, Shenzhen, China). The dumbbell-shaped specimens for the tensile tests were prepared according to ASTM D-638 (type I) at a crosshead speed of $50 \mathrm{~mm} \mathrm{~min}{ }^{-1}$, with a gauge length of $50 \mathrm{~mm}$. Static flexural tests were conducted using a three-point bending set up according to ASTM D-790 under a crosshead speed of $2 \mathrm{~mm} \mathrm{m^{-1 }}$, and its dimensions were $127 \times 12.7 \times 3.0 \mathrm{~mm}^{3}$ (length $\times$ width $\times$ thickness). The notched Izod impact strength of the specimens was tested according to ASTM D-256 using an impact testing machine (Zwick/Roell HIT 25P, Zwick Roell Testing Machine Pvt. Ltd.). The dimensions of the specimens were $63.5 \times 12.7 \times 3.0 \mathrm{~mm}^{3}$ with a V notch. All mechanical tests were carried out at room temperature. For each specimen, a minimum of seven measurements were conducted, and average results were reported.

2.3.2 SEM. SEM measurement was conducted to investigate the interfacial adhesion between the GF and PP matrix and

Table 1 Components and contents of various PP composites ${ }^{a}$

\begin{tabular}{lllll}
\hline Sample & & & & \\
\hline PP & TMP-6 (wt\%) & TMA-3 (wt\%) & GF (wt\%) & $\begin{array}{l}\text { PP-MA } \\
(w t \%)\end{array}$ \\
GF/PP & N/A & N/A & N/A & N/A \\
TMP-6/PP & N/A & N/A & 20 & 2 \\
& 0.1 & N/A & N/A & N/A \\
& 0.2 & N/A & N/A & N/A \\
TMA-3/PP & 0.3 & N/A & N/A & N/A \\
& N/A & 0.1 & N/A & N/A \\
& N/A & 0.2 & N/A & N/A \\
TMP-6/GF/PP & N/A & 0.3 & N/A & 2 \\
& 0.1 & N/A & 20 & 2 \\
& 0.3 & N/A & 20 & 2 \\
TMA-3/GF/PP & N/A & N/A & 20 & 2 \\
& N/A & 0.1 & 20 & 2 \\
& N/A & 0.2 & 20 & 2
\end{tabular}

${ }^{a}$ N/A not applicable. the supermolecular structure of $\alpha$-nucleated PP. A fieldemission scanning microscope (FE-SEM, ZEISS SUPRA 55VP) was used at an acceleration voltage of $20 \mathrm{kV}$. The fracture surfaces produced during the tensile tests were chosen to observe the interfacial adhesion and the morphology of the modified PP. To clearly observe the microscopic structure of the PP composites, each sample was treated at $35{ }^{\circ} \mathrm{C}$ for $30 \mathrm{~h}$ with an etchant containing $1.3 \mathrm{wt} \%$ potassium permanganate $\left(\mathrm{KMnO}_{4}\right), 32.9 \mathrm{wt} \%$ concentrated sulfuric acid $\left(\mathrm{H}_{2} \mathrm{SO}_{4}, 98 \%\right)$, and $65.8 \mathrm{wt} \%$ concentrated phosphoric acid $\left(\mathrm{H}_{3} \mathrm{PO}_{4}\right)$ according to a reported procedure. ${ }^{30}$ All the samples were coated with a thin layer of gold before SEM observation.

2.3.3 Wide-angle XRD (WAXD) characterization. The crystal structure of the PP composite was investigated using an XRD (D8-ADVANCE, Bruker AXS, Germany). The samples were measured in the diffraction angle $(2 \theta)$ range of $10-30^{\circ}$ at a scanning rate of $0.02^{\circ} \mathrm{s}^{-1}$. The entire experiments were conducted using a Cu- $\mathrm{K}_{\alpha}(\lambda=0.154 \mathrm{~nm})$ source at a generator voltage of $40 \mathrm{kV}$ and a current of $40 \mathrm{~mA}$.

2.3.4 DSC characterization. The melting and nonisothermal crystallization behaviors of the PP composites were studied using a thermal analysis apparatus (Nietzsche, STA449F3, Germany) under a nitrogen atmosphere. The heat flow and temperature calibration were carried out with indium as a standard medium before carrying out the test. Measurements were conducted with 5-8 $\mathrm{mg}$ samples, which were first heated from room temperature to $200{ }^{\circ} \mathrm{C}$ at a heating rate of $10{ }^{\circ} \mathrm{C} \min ^{-1}$ and then held at $200{ }^{\circ} \mathrm{C}$ for $5 \mathrm{~min}$ to erase the thermal and mechanical history. Subsequently, the melted sample was cooled down to $50{ }^{\circ} \mathrm{C}$ at a cooling rate of $5{ }^{\circ} \mathrm{C} \mathrm{min}^{-1}$. After reaching the minimum temperature, the sample was reheated to $200{ }^{\circ} \mathrm{C}$ at a heating rate of $10{ }^{\circ} \mathrm{C} \mathrm{min}{ }^{-1}$. From the DSC curves, the degree of crystallinity $\left(X_{\mathrm{c}}\right)$ of the sample was calculated using the following equation: ${ }^{31}$

$$
X_{\mathrm{c}}=\frac{\Delta H_{\mathrm{m}}}{\Delta H_{\mathrm{m}}^{0} w_{\mathrm{m}}}
$$

where $\Delta H_{\mathrm{m}}$ represents the apparent heat of fusion of the composite and is obtained by integration from the base line to the DSC thermogram. $w_{\mathrm{m}}$ is the weight fraction of the PP matrix, and $\Delta H_{\mathrm{m}}^{0}$ is the melting enthalpy of a $100 \%$ crystalline PP and taken as $177 \mathrm{~J} \mathrm{~g}^{-1}$. $^{31}$

2.3.5 Polarized optical microscopy (POM). Crystallization morphologies of the pure PP and nucleated PP samples, which were first heated to $200{ }^{\circ} \mathrm{C}$ and held for $5 \mathrm{~min}$ to erase thermal history, were characterized using a polarized optical microscope sandwiched between two microscope cover slips. Then, the sample was rapidly cooled down to a predetermined crystallization temperature of $135{ }^{\circ} \mathrm{C}$ and kept for $20 \mathrm{~min}$ to isothermally complete the crystallization process. The morphologies were obtained and magnified by 400 times.

2.3.6 HDT measurement. HDT was tested in a three-point mode according to the ASTM D-648 standard using a Vicat/HDTTester Compact 6 (Coesfeld GmbH \& Co. KG, Germany). Before testing, all the specimens were kept at $23 \pm 2{ }^{\circ} \mathrm{C}$ and $50 \pm 5 \% \mathrm{RH}$ for $48 \mathrm{~h}$. The samples were subjected to deflection by $0.25 \mathrm{~mm}$ under a surface load of $0.455 \mathrm{MPa}$ and a heating rate of $2{ }^{\circ} \mathrm{C} \mathrm{min}^{-1}$. 


\section{Results and discussion}

\subsection{Mechanical properties}

PP is one of the most important commercial versatile thermoplastic polymers due to its good comprehensive properties. ${ }^{6}$ However, for high-performance applications, its mechanical and thermal properties still need to be improved. Thus, it is essential to investigate the combined effect of $\alpha$-NAs and GFs on the mechanical properties of the PP composites. Tensile strength, flexural modulus, and impact strength of neat PP and PP modified with GFs, TMP-6, TMA-3, TMP-6/GF, and TMA-3/GF with different loadings of $\alpha$-nucleating agents are presented in Fig. 2a-c, respectively. The mechanical properties, especially the tensile strength and flexural modulus, of PP reinforced with $20 \mathrm{wt} \%$ GFs were improved as compared to that of neat PP, which increased by $87.06 \%$ and $158.25 \%$, respectively. These results were in agreement with the study of short-glass-fiberreinforced polypropylene reported by Flores et $a .^{32}$ The enhancement was attributed to the fact that the high-strength GF fillers not only were beneficial for the external load transfer from the PP matrix to GFs, but also could act as a crack stopper. The high-strength GFs could bear the main stress under external load and act as a support matrix to effectively dissipate the external load, ${ }^{33}$ thus improving the mechanical properties of the PP composites.

To investigate the effect of the type and content of $\alpha$-NAs on the mechanical properties of $\mathrm{PP}$, the mechanical properties of PP and nucleated PP were compared. As expected, both the tensile strength and the flexural modulus (stiffness) of nucleated PP (TMP-6 or TMA-3 individually) significantly increased, whereas its impact strength (toughness) drastically decreased. First, the tensile strength and flexural modulus of PP were enhanced with the increasing amount of $\alpha$-NAs until a maximum loading of $0.2 \mathrm{wt} \%$. Second, the tensile strength and flexural modulus dropped when the content of $\alpha$-NAs was further increased to $0.3 \mathrm{wt} \%$, where the stiffness of TMP- 6 and TMA-3-nucleated PP showed a slight difference. The reduction of stiffness could be ascribed to the possibility that the excessive amount of NAs agglomerated due to the strong hydrogen bonding. For both TMP-6/PP and TMA-3/PP composites, the flexural modulus almost doubled as compared to that of neat PP at $0.2 \mathrm{wt} \% \alpha$-NAs. The enhanced stiffness was attributed to the nucleating effect on the PP crystallization process as the formation of the crystalline regions was favorable for the enhancement of the modulus of semi-crystalline polymers. It was noted that the impact strength of PP nucleated by TMP- 6 or TMA-3 decreased, but the decrease was more obvious for TMP-6 than that for TMA-3. Moreover, it was observed that TMP-6 showed a slightly higher improvement than TMA-3.

To achieve improved and balanced mechanical properties of the PP composite, the incorporation of both $\alpha$-NAs (TMP- 6 and TMA-3) and GFs into the PP matrix could be an alternative and promising approach. As clearly shown in Fig. 2a-c, compared with that of the TMP-6/PP and TMA-3/PP composites, the stiffness and toughness of TMP-6/GF/PP and TMA-3/GF/PP were improved. Especially, there was a notable increase in toughness. As shown in Fig. 2a, for TMP-6/GF/PP and TMA-3/GF/PP composites, the tensile strength increased with the content of $\alpha$-NAs and then slightly decreased. With $0.2 \mathrm{wt} \% \alpha$-NA loading, the tensile strength reached the maximum values of 41.9 and $37.8 \mathrm{MPa}$. There was an increase of $24.9 \%$ and $12.7 \%$ in the tensile strength of TMP-6/GF/PP and TMA-3/GF/PP composites, respectively, as compared to that of the GF/PP composite. Generally, the tensile strength strongly depends on the weakest part of composites. The results indicated an improved interfacial adhesion between the GFs and PP matrix. The promoted interfacial interaction was observed in the SEM images (Fig. 6). The flexural modulus displayed a similar tendency as the tensile
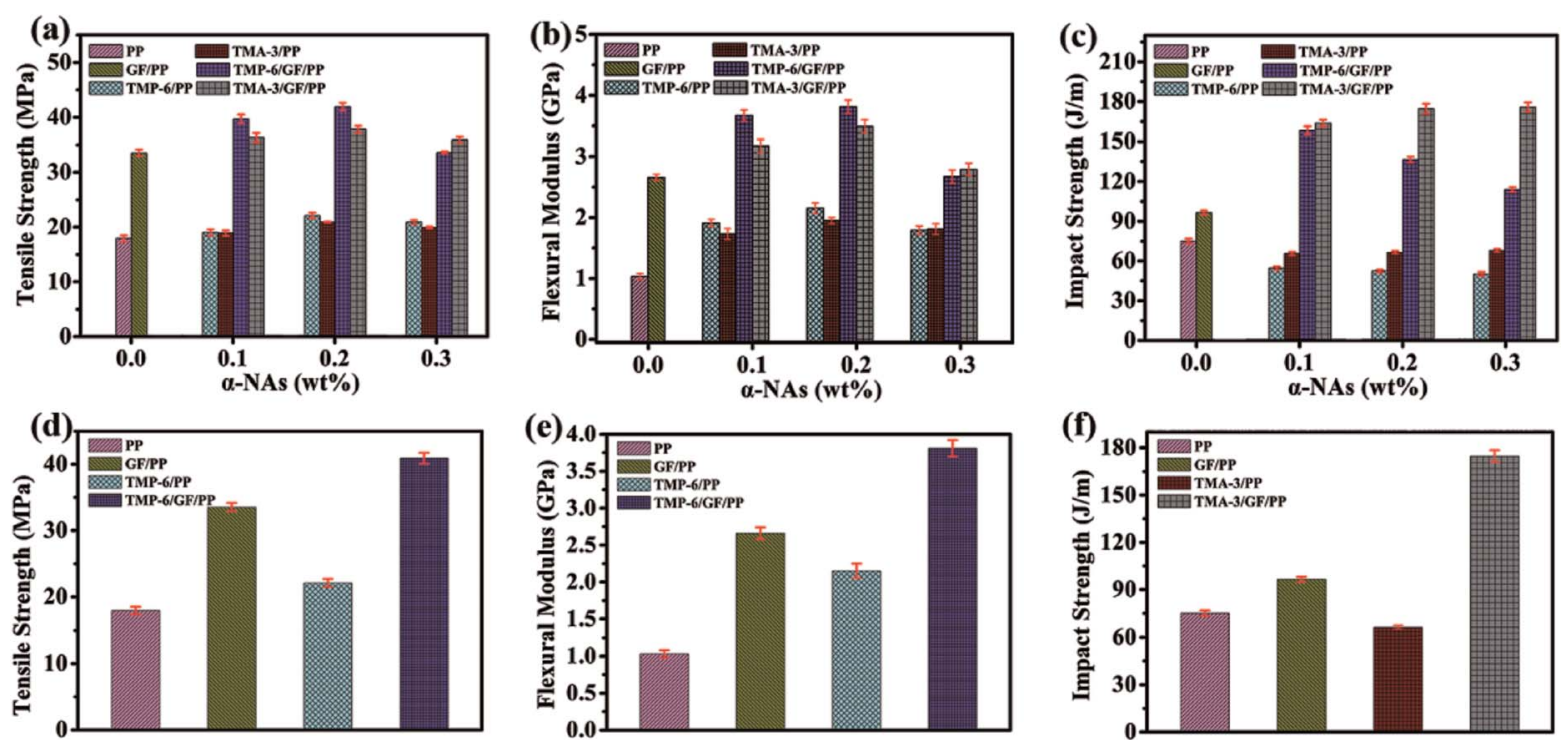

Fig. 2 Mechanical properties of the PP composites with different amounts of $\alpha$-nucleating agents: (a) tensile strength, (b) flexural modulus, and (c) impact strength. The (d) tensile strength, (e) flexural modulus, and (f) impact strength of the PP composites with 0.2 wt\% TMA-3 and TMP-6. 
strength, and the optimum values for TMP-6/GF/PP and TMA-3/ $\mathrm{GF} / \mathrm{PP}$ were 3.81 and $3.49 \mathrm{GPa}$, respectively. These results revealed that there was a synergistic effect between GFs and $\alpha$ NAs on enhancing the tensile and flexural properties of the PP composites. With regard to the impact strength, TMP-6/GF/PP and TMA-3/GF/PP exhibited a significantly higher strength than both TMP-6/PP and TMA-3/PP composites; this was ascribed to the fact that GFs could absorb a part of the impact energy and prevent crack propagation. Specifically, the impact strength of TMP-6/GF/PP decreased, whereas that of TMA-3/GF/ PP slightly increased with the addition of $\alpha$-NAs. Overall, it was found that GF-reinforced $\alpha$-PP offered better stiffness-toughness balance than the conventional approaches using $\alpha$-nucleating agents or via the addition of glass fibers individually. The presence and combination of GFs and $\alpha$-NAs could also lead to better interfacial stress transfer between GFs and the PP matrix. The improvement in the mechanical properties of PP composites could be ascribed to the combined effect between GFs and $\alpha$-NAs. The mechanically strong GFs acted as a support matrix to effectively dissipate external load. Furthermore, the better interfacial adhesion between GFs and the polymer matrix as compared to that of the GF/PP composite was also another important factor. As shown in Fig. $2 \mathrm{~d}$ and e, the TMP-6/GF/PP composite exhibited highest stiffness, and the tensile strength and flexural modulus were improved by $134 \%$ and $270 \%$ as compared to those of neat PP, respectively; on the other hand, TMA-3/GF/PP displayed highest impact strength that increased by $133 \%$ as compared to that of neat PP, as clearly shown in Fig. 2f. To better understand the mechanism of enhancement of the mechanical properties of the PP composites modified by GFs and $\alpha$-NAs, DSC, XRD, and SEM were employed to characterize and compare the mechanical properties of the neat PP and modified PP composites.

\subsection{Crystallization and melting behavior of the PP composites}

The melting behavior of the samples was monitored by DSC to investigate the changes in the crystalline structure, and the crystallization behavior of the PP composites is presented in Fig. 3a and b. For better comparison, the melting behaviors of $\mathrm{PP}$ and GF/PP are also shown. It is apparent, as shown in Fig. 3a and $\mathrm{S} 1, \dagger$ that all the samples exhibit one single endothermic melting peak at around $167{ }^{\circ} \mathrm{C}$, which is associated with the melting of $\alpha$-crystals. ${ }^{34}$ These results further demonstrated that only one $\alpha$-crystal was formed during the melting process of materials. This indicated that TMP- 6 and TMA- 3 studied herein acted as $\alpha$-NAs of PP. The melting temperatures $\left(T_{\mathrm{m}}\right)$ of modified PP were slightly higher than those of PP; the stronger the nucleating ability, the higher the $T_{\mathrm{m}} \cdot{ }^{\mathbf{8} 35}$ The modified PP composites demonstrated increased crystallization temperature; thus, their melting temperature increased. Moreover, these results revealed that GFs could act like an NA to some extent in the GF/PP composite and facilitate the formation of an $\alpha$-form crystalline structure. , $^{\mathbf{3 5}}$

The crystallization process plays an essential role in the properties of semi-crystalline polymers. It is important to
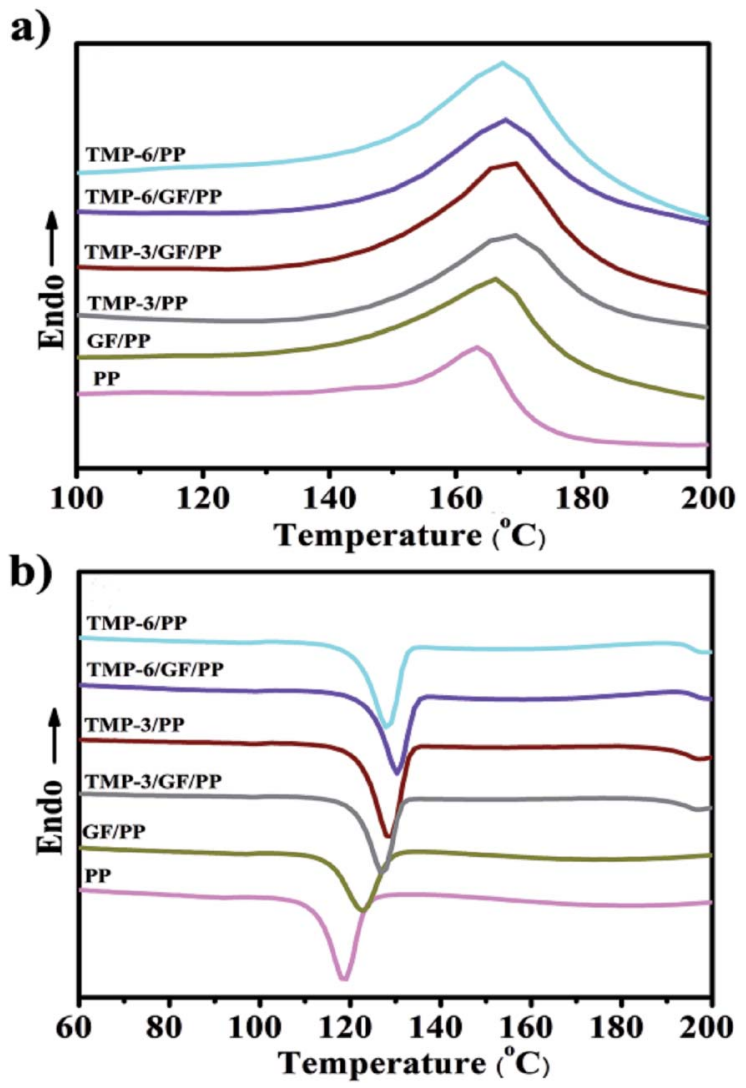

Fig. 3 Melting and crystallization behavior of various composites measured via of DSC: (a) melting at $10{ }^{\circ} \mathrm{C} \mathrm{min}^{-1}$ and (b) cooling at $5{ }^{\circ} \mathrm{C} \mathrm{min}-1$ (the concentration of TMP- 6 and TMA-3 is $0.2 \mathrm{wt} \%$ ).

elucidate the influence of $\alpha$-NAs and GFs on the crystallization behavior of PP (Fig. 3b and S2 $\dagger$ ). The thermal parameters, such as crystallization temperature $\left(T_{\mathrm{c}}\right)$, degree of crystallinity $\left(X_{\mathrm{c}}\right)$, and half time of crystallization $\left(t_{1 / 2}\right)$, obtained from the DSC analysis of the PP composites are summarized in Table 2. $T_{\mathrm{c}}$ of $\mathrm{GF} / \mathrm{PP}$ was $122.7^{\circ} \mathrm{C}$, which was higher than that of pure PP. This result suggested that the GFs had a positive effect on the promotion of the crystallization of PP and could act as nucleating agents. As expected, the introduction of TMP- 6 or TMA-3 into the PP matrix induced an increase in $X_{\mathrm{c}}$ and $T_{\mathrm{c}}$; this indicated that both TMP- 6 and TMA- 3 were effective $\alpha$-NAs and could facilitate the crystallization of the polymer chains and accelerate the crystallization rate of PP. The $T_{\mathrm{c}}$ of TMP-6/GF/PP was nearly equivalent to that of TMP-6/PP, and a similar tendency was observed for TMA-3/PP and TMA-3/GF/PP. These results indicated that $\alpha$-NAs played a dominated role in the $T_{\mathrm{c}}$. These results were consistent with those observed in WAXD. It is well known that the higher the $T_{\mathrm{c}}$, the lower the barrier of nucleation. Results suggested that both GFs and $\alpha$-NAs could not only induce the nucleation, but also promote the crystallization of PP. However, it could be clearly seen that the nucleation efficiency of $\alpha$-NAs (TMP-6 and TMA-3) was much higher than that of GFs. With respect to the nucleating efficiency (NE), which is commonly used to investigate the nucleation behavior of polymer composites, the NE can be obtained using the following equation: ${ }^{36}$ 
Table 2 Crystallization behavior of various composites ${ }^{a}$

\begin{tabular}{lllll}
\hline Sample & $\begin{array}{l}\alpha \text {-NAs } \\
\text { content }(w t \%)\end{array}$ & $T_{\mathrm{c}}\left({ }^{\circ} \mathrm{C}\right)$ & $X_{\mathrm{c}}(\%)$ & $\begin{array}{l}\text { Crystallization } \\
\text { rate } t_{1 / 2}(\mathrm{~s})\end{array}$ \\
\hline $\mathrm{PP}$ & 0 & 118.5 & 50.7 & 198.1 \\
GF/PP & 0 & 122.7 & 48.2 & 168.1 \\
TMP-6/PP & 0.1 & 127.5 & 54.5 & 131.9 \\
& 0.2 & 128.1 & 54.7 & 128.4 \\
& 0.3 & 128.3 & 55.1 & 128.6 \\
TMA-3/PP & 0.1 & 123.6 & 53.8 & 155.7 \\
& 0.2 & 128.5 & 54.9 & 133.2 \\
TMP-6/GF/PP & 0.3 & 127.7 & 54.6 & 130.2 \\
& 0.1 & 128.4 & 55.2 & 134.4 \\
& 0.2 & 130.1 & 57.1 & 123.2 \\
TMA-3/GF/PP & 0.1 & 129.6 & 56.7 & 117.3 \\
& 0.2 & 124.5 & 56.7 & 153.6 \\
& 0.3 & 127.1 & 54.8 & 133.8 \\
& & 127.2 & 54.5 & 132.6
\end{tabular}

${ }^{a} T_{\mathrm{c}}$, crystallization temperature; $X_{\mathrm{c}}$, degree of crystallinity; and $t_{1 / 2}$, half time of crystallization.

$$
\mathrm{NE} \%=100 \% \frac{T_{\mathrm{cNA}}-T_{\mathrm{c} 1}}{T_{\mathrm{c} 2 \max }-T_{\mathrm{c} 1}}
$$

where $T_{\mathrm{cNA}}, T_{\mathrm{c} 1}$, and $T_{\mathrm{c} 2 \mathrm{max}}$ represent the peak temperature of the nucleated, non-nucleated, and self-nucleated polymer, respectively. In some cases, $T_{\mathrm{c} 1}$ and $T_{\mathrm{c} 2 \mathrm{max}}$ were constant. Therefore, it can be considered that the higher the $T_{\text {cNA }}$, the better the NE. From Table 2, it can be seen that the $T_{\mathrm{cNA}}$ of PP nucleated with $\alpha$-NAs was higher than that of PP reinforced by GFs; thus, the NE of PP modified by $\alpha$-NAs was greater than that of GF-reinforced PP.

Isothermal crystallization of PP and its composites can be described by the Avrami equation. The relative degree $\left(X_{t}\right)$ of polymers at time $t$ can be calculated using the following equation: ${ }^{37}$

$$
X_{t}=\frac{X_{t}(t)}{X_{t}(\infty)}=\frac{\int_{0}^{t}(\mathrm{~d} H-\mathrm{d} t) \mathrm{d} t}{\int_{0}^{\infty}(\mathrm{d} H-\mathrm{d} t) \mathrm{d} t}
$$

where $\mathrm{d} H / \mathrm{d} t$ is the heat flow rate in the process of isothermal crystallization at time $t$ and $X_{t}(t)$ and $X_{t}(\infty)$ are the absolute degrees of crystallinity at time $t$ and total crystallization time, respectively. Time $t$ was calculated from the moment when the material was cooled down to a certain crystallization temperature. The variation in the relative degree of crystallinity $\left(X_{t}\right)$ values as a function of crystallization time $(t)$ is shown in Fig. 4 . The evolution of relative crystallinity with crystallization time at various temperatures showed S-shaped curves, which were consistent with the typical curves of the nucleation and growth processes of polymers. The half time of crystallization $\left(t_{1 / 2}\right)$ is commonly used to evaluate the rate of crystallization of the polymers, which is the time taken for $50 \%$ total crystallization. It is known that a shorter $t_{1 / 2}$ is associated with a higher crystallization rate. From Table 2, it can be observed that the incorporation of GFs and $\alpha$-NAs (TMP- 6 and TMA-3), both separately and in combination, decreases the $t_{1 / 2}$ values of modified PP; this indicates the increased crystallization rate.

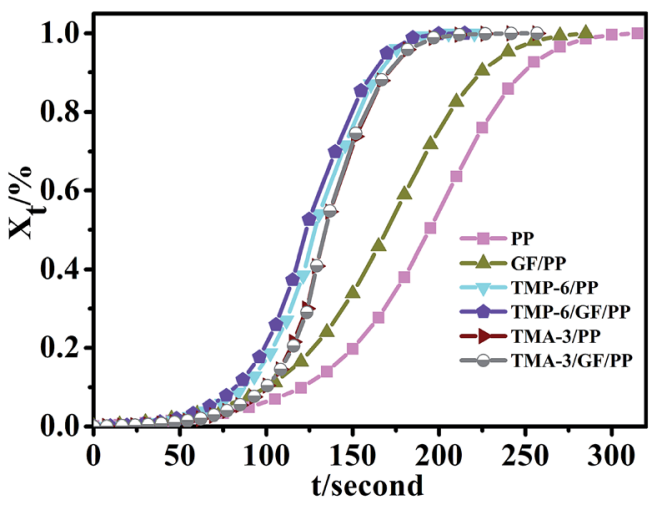

Fig. 4 Curves of relative degree of crystallization $X_{t}$ versus time $t$ (the concentration of TMP- 6 and TMA-3 is $0.2 \mathrm{wt} \%$ ).

Furthermore, it was also found that TMP-6 was more effective than TMA-3 in terms of the crystallization rate. However, the presence of GFs in TMP-6/GF/PP and TMA-3/GF/PP had a little effect on $t_{1 / 2}$.

\subsection{Crystal structure}

To further investigate the change in the crystal structure of PP composites, WAXD measurements were conducted. The results are shown in Fig. 5 and S3. $\dagger$ As observed, the virgin PP sample exhibited the characteristic diffraction peaks of $\alpha$-PP at the $2 \theta$ of $14.2^{\circ}, 16.9^{\circ}$, and $18.5^{\circ}$, corresponding to the crystal planes $(110)$, (040), and (130), respectively. ${ }^{38}$ The same diffraction peaks also appeared in the diffraction pattern of TMP-6/PP, TMA-3/PP, TMP-6/GF/PP, and TMA-3/GF/PP composites. The results were consistent with the crystallization temperature obtained from the DSC crystallization curves. The crystallization temperatures of TMP-6/PP and TMA-3/PP were found to be $127.7{ }^{\circ} \mathrm{C}$ and $128.5{ }^{\circ} \mathrm{C}$, respectively, whereas, for neat $\mathrm{PP}$, it was $118.5{ }^{\circ} \mathrm{C}$. These results indicated that the presence of $\alpha$-NAs (TMP-6/PP or TMA-3/PP) only changed the crystallization rate of PP. Moreover, the crystalline structure of $\mathrm{PP}$ was retained since no new reflections related to other crystal phases were observed. As for

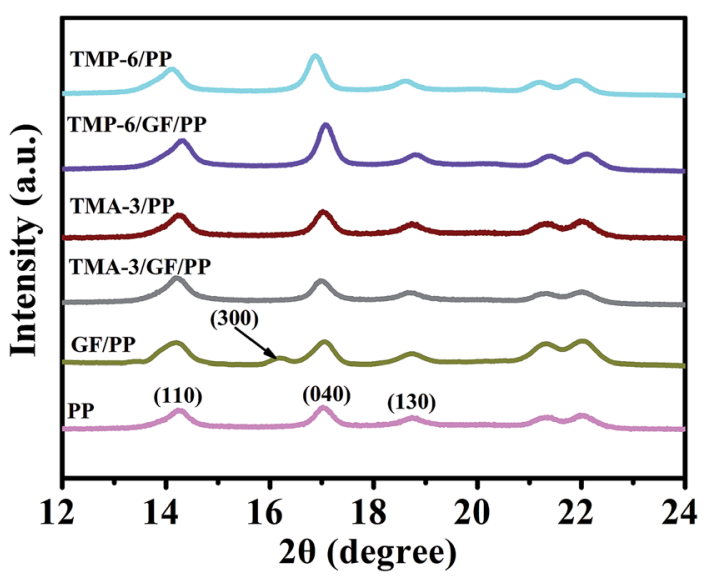

Fig. 5 WAXD patterns of various composites (the concentration of TMP- 6 and TMA-3 is $0.2 \mathrm{wt} \%$ ). 
GF/PP, a new peak at around $16.4^{\circ}$ appeared, which was attributed to the crystal plane (300), suggesting that small percentage of $\beta$-crystals existed. Results showed that $\beta$-crystals were absent in the DSC plot; this was due to phase transformation from $\beta$ crystals to $\alpha$-crystals during melting, cooling, and melting process. ${ }^{39}$ This implied that GF could induce the formation of $\beta$ crystals of PP. However, no $\beta$-crystals appeared in the PP matrix upon combining the GF and $\alpha$-NAs. This could be due to the competitive nucleation between GF and $\alpha$-NAs, whereas $\alpha$-NAs played a dominating role in the crystallization process.

The crystal morphologies of pure PP and nucleated PP were investigated by POM. In Fig. 6, it was observed that the morphology of pure PP showed spherulite appearance with large spherulite sizes. With the addition of $\alpha$-NAs (TMP- 6 or TMA-3), more nucleation sites were formed and thus much smaller crystal size was observed; this indicated that TMP- 6 or TMA-3 indeed acted as an effective nucleating agent in the PP matrix. The observations were in agreement with the results of DSC and mechanical performance, where the crystallization temperatures increased; the mechanical properties of nucleated PP were improved as compared to those of pure PP.

\subsection{SEM characterization}

As abovementioned, with the addition of $\alpha$-NAs and GFs, both the interfacial adhesion between PP and GFs and mechanical properties of the PP composites improved. To better understand the enhancement mechanism, SEM was employed to analyze the tensile-fracture surfaces of various composites, as shown in Fig. 7. The morphologies of neat PP, TMP-6/PP, and TMA-3/PP composites are shown in Fig. 7a-c, respectively. The neat PP showed a typical fracture type and the surface was relatively smooth. However, the fracture section of TMP-6/PP and TMA-3/ PP composites became uneven; this indicated that $\alpha$-modification increased the content of crosshatching, decreased the crystal

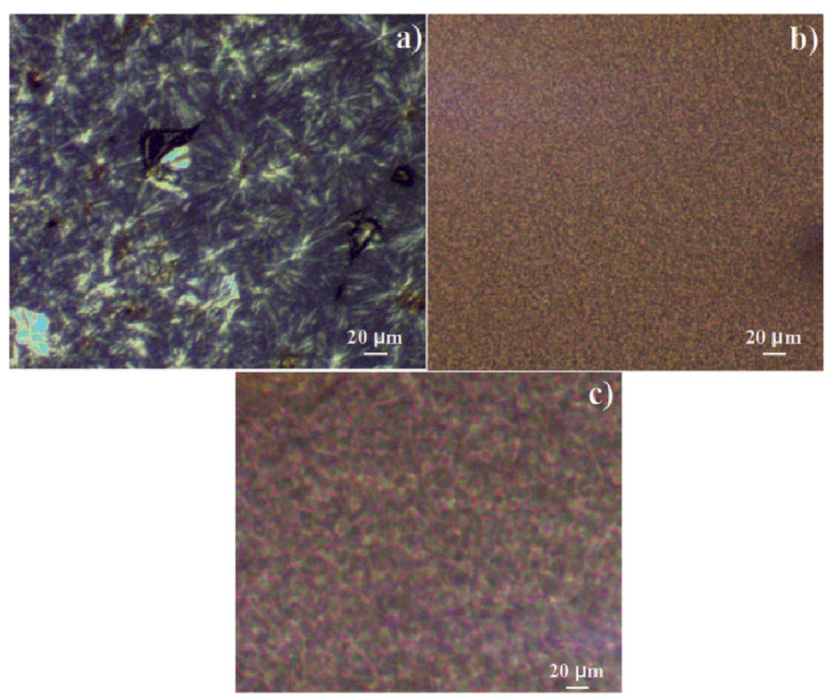

Fig. 6 Polarized optical microscopic morphologies of (a) pure PP, (b) TMP-6/PP, and (c) TMA-3/PP (the concentration of TMP- 6 and TMA-3 is $0.2 \mathrm{wt} \%$ ). slip ability, and consequently, improved the stiffness, whereas the toughness decreased. The results indicated that TMP- 6 was more effective than TMA-3 in enhancing the stiffness, whereas TMA-3 was more favorable for absorbing more impact energy.

At a low magnification in Fig. 7d and e, the surfaces of pullout fibers can be clearly seen. The fractured surfaces were different for each PP composite. In the case of GF/PP, on the surface of GFs, there was no obvious PP matrix sticking on them. This result demonstrated that the adhesion interaction between polymer matrix and GFs was weak and the compatibility was poor. ${ }^{40}$ On the other hand, for TMP-6/GF/PP and TMA3/GF/PP composites, there were abundant PP matrices sticked on the surface of GFs (Fig. 7e and f). These results can be observed more clearly at a higher magnification (Fig. 7d1-f1). The abovementioned findings further implied a strong adhesion between GFs and PP in TMP-6/GF/PP and TMA-3/GF/PP composites. These were convincing evidence that the interfacial adhesion was significantly improved. Interestingly, it was observed that the interfacial adhesion of TMP-6/GF/PP was stronger than that of TMA-3/GF/PP.

Based on the abovementioned discussion, the major roles of the filler of GFs in improving the toughness of TMP-6/GF/PP and TMA-3/GF/PP could be ascribed to the following mechanism: first, the crack tips were blunted by interconnected GF network before polymer matrix started to deflect. As a consequence, the PP matrix tended to tilt, twist or bifurcate along the boundary of GFs under mixed modes. ${ }^{41}$ Under these mixed conditions, due to the collapse of the composites, more absorption of energy was required than that in the case of a normal polymer. Second, the interphase between GFs and matrix would debond during the process of crack tip blunting, and strong interfacial bonding and adhesion were beneficial to both composites (Fig. 7e1 and f1). It has been demonstrated that the interfacial debonding would result in an enhancement of energy absorption in the composite. ${ }^{\mathbf{4 2}}$

\subsection{Heat distortion temperature (HDT)}

The influence of $\alpha$-NAs and GFs on the thermomechanical behavior of PP was investigated. HDT is a temperature at which a polymeric material deforms under a constant load.

This property is a critical parameter for product design and engineering and manufacturing process of products. High HDT indicates that composites still possess excellent performance under high-temperature conditions. Both GFs and aromatic phosphate salt have been reported to improve the HDT of PP. ${ }^{\mathbf{8 , 3 1}}$ However, to the best of our knowledge, the HDT of PP composites modified by combining GFs and $\alpha$-NAs has never been reported. The HDT temperatures of different samples are summarized in Fig. 8. The HDT of GF/PP was $145{ }^{\circ} \mathrm{C}$, higher than that of neat PP. Both TMP-6/PP and TMA-3/PP showed only a slight increase in the HDT as compared to the neat PP; this indicated that the incorporation of GFs and $\alpha$-NAs (TMP- 6 and TMA-3) could lead to an increase in HDT of PP. In the case of the TMP-6/GF/PP and TMA-3/GF/PP composites, they were more or less the same value regardless of the concentration of NAs, being approximately $143-148{ }^{\circ} \mathrm{C}$ and $141-146^{\circ} \mathrm{C}$, respectively. 

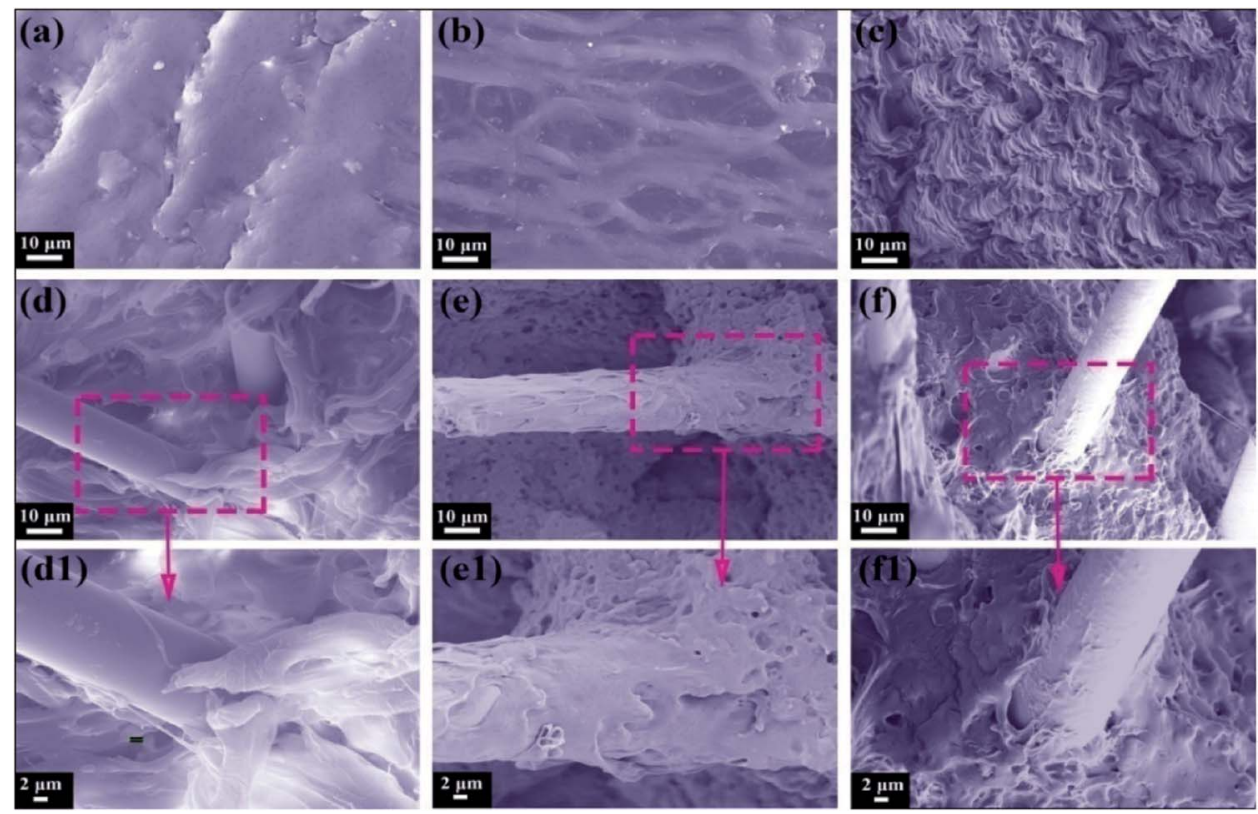

Fig. 7 SEM images of (a) neat PP, (b) TMP-6/PP, (c) TMA-3/PP, (d) GF/PP, (e) TMP-6/GF/PP, and (f) TMA-3/GF/PP (the concentration of TMP-6 and TMA-3 is 0.2 wt\%). The images with higher magnification (d1), (e1), and (f1) represent a magnified area of the fracture surface.

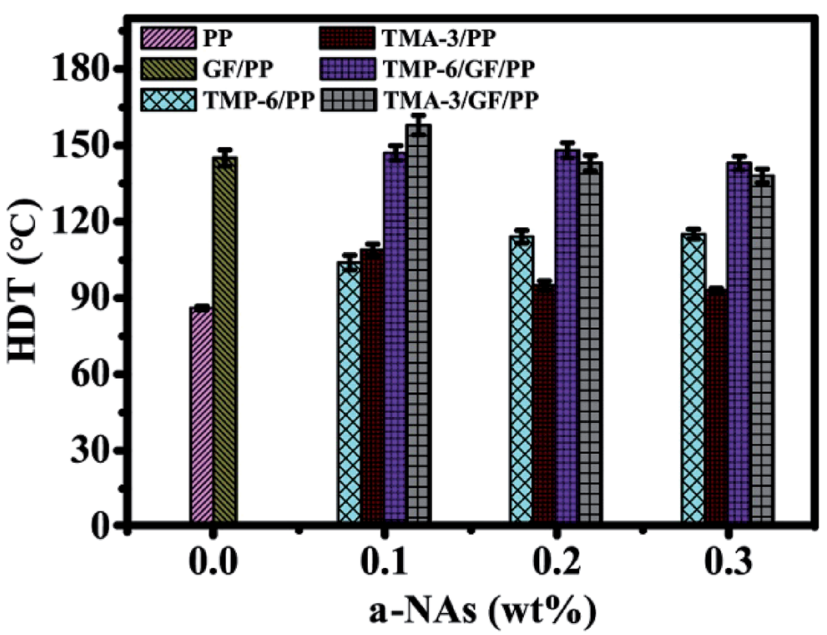

Fig. 8 Heat distortion temperature of various PP composites.

This tendency was very similar to that of the flexural modulus, and the measurements were also conducted in a three-point bending mode like the flexural property test. Several factors, such as the degree of crystallinity and the stiffness, were reported to have an influence on the HDT of polymers. ${ }^{37}$ The incorporation of GFs could lead to a significant improvement in stiffness, hence increasing the HDT of the PP matrix. Both the $X_{\mathrm{c}}$ and stiffness achieved a maximum value at $0.2 \mathrm{wt} \% \alpha$-NAs at which TMP-6/PP and TMA-3/PP presented the best HDT. The addition of $\alpha$-NAs increased the crystallinity of PP and led to an enhancement in the heat distortion temperature. Furthermore, it was important to notice that the GFs played a dominated role in the HDT of GF/PP. The results were consistent with the synergetic behavior of flexural properties.

\section{Conclusions}

In this study, the effect of the incorporation of $\alpha$-NAs (TMP-6 and TMA-3) and GFs, both individually and in combination, on the morphology, crystal structure, mechanical properties, and HDT of polypropylene was investigated. It was found that the incorporation of the GFs could increase the crystallization temperature and the crystallization rate of the PP composites and their thermomechanical property. The variation in the concentration of $\alpha$-NAs could influence the mechanical performance. PP composites exhibited the optimum comprehensive performance with $0.2 \mathrm{wt} \% \alpha$-NAs loading. Among all the composites, TMP-6/GF/PP showed the best stiffness (tensile strength and flexural modulus), and the TMA-3/GF/PP displayed the best toughness.

Compared with that of the neat $\mathrm{PP}$ and GF/PP, the impact strength of TMA-3/GF/PP (0.2 wt $\%$ TMA-3) increased by $133 \%$ and $104 \%$, respectively. The reinforcement mechanism was proposed due to both enhanced interfacial adhesion and the combined effect of GFs and $\alpha$-NAs. Our study provided a feasible and alternative approach to obtain PP composites with comprehensive high performance, in particular balanced tensile, flexural, and impact properties.

\section{Conflicts of interest}

There are no conflicts to declare.

\section{Acknowledgements}

This work was financially supported by National Natural Science Foundation of China (21472235, 21464015), Xinjiang Distinguished Youth Scholar Program (qn2015jq012) and "One 
Thousand Talents" Program of China (Y32H291501), the China National high technology research and development program (863 program) (SS2015AA034003), the China Postdoctoral Science Foundation (154200) and the STS program of Chinese Academy of Sciences (2017).

\section{Notes and references}

1 M. Gahleitner, C. Tranninger and P. Doshev, J. Appl. Polym. Sci., 2013, 130, 3028-3037.

2 J. Varga, J. Mater. Sci., 1992, 27, 2557-2579.

3 B. Lotz, J. Wittmann and A. Lovinger, Polymer, 1996, 37, 4979-4992.

4 J. Varga, J. Macromol. Sci., Part B: Phys., 2002, 41, 1121-1171. 5 A. Menyhárd, M. Gahleitner, J. Varga, K. Bernreitner, P. Jääskeläinen, H. Øysæd and B. Pukánszky, Eur. Polym. J., 2009, 45, 3138-3148.

6 T. Dobreva, J. López-Majada, J. Perena, E. Perez and R. Benavente, J. Appl. Polym. Sci., 2008, 109, 1338-1349.

7 M. Dobrzyńska-Mizera, M. Dutkiewicz, T. Sterzyński and M. L. Di Lorenzo, Eur. Polym. J., 2016, 85, 62-71.

8 Y. F. Zhang and Z. Xin, J. Appl. Polym. Sci., 2006, 100, 48684874.

9 Y. Jin, A. Hiltner and E. Baer, J. Appl. Polym. Sci., 2007, 105, 3260-3273.

10 S. C. Zhao and Z. Xin, J. Appl. Polym. Sci., 2009, 112, 14711480.

11 Z. Cai, S. C. Zhao, B. X. Shen and Z. Xin, J. Appl. Polym. Sci., 2010, 116, 792-800.

12 A. Thierry, C. Straupé, J. C. Wittmann and B. Lotz, Macromol. Symp., 2006, 241, 103-110.

13 G. Coulon, G. Castelein and C. G'sell, Polymer, 1999, 40, 95110.

14 P. Tordjeman, C. Robert, G. Marin and P. Gerard, Eur. Phys. J. E: Soft Matter Biol. Phys., 2001, 4, 459-465.

15 K. Rhee, S. Lee and J. Lee, Mater. Sci. Technol., 2005, 21, 743748.

16 C. Roux, J. Denault and M. F. Champagne, J. Appl. Polym. Sci., 2000, 78, 2047-2060.

17 S. Patcharaphun and G. Mennig, Polym. Compos., 2005, 26, 823-831.

18 J. Thomason and M. Vlug, Composites, Part A, 1997, 28, 277288.

19 J. Thomason, Composites, Part A, 2002, 33, 1641-1652.

20 L. T. Drzal, M. J. Rich and P. F. Lloyd, J. Adhes., 1983, 16, 130.
21 J.-K. Kim and Y.-W. Mai, Compos. Sci. Technol., 1991, 41, 333378.

22 D. A. Jesson and J. F. Watts, Polym. Rev., 2012, 52, 321-354.

23 J. L. Thomason and G. Schoolenberg, Composites, 1994, 25, 197-203.

24 G. J. Luo, W. Z. Li, W. B. Liang, G. G. Liu, Y. Ma, Y. H. Niu and G. X. Li, Composites, Part B, 2017, 111, 190-199.

25 D. Bikiaris, P. Matzinos, A. Larena, V. Flaris and C. Panayiotou, J. Appl. Polym. Sci., 2001, 81, 701-709.

26 S. C. Tjong, S.-A. Xu, R. K.-Y. Li and Y.-W. Mai, Compos. Sci. Technol., 2002, 62, 831-840.

27 X. X. Chen, L. Yuan, Z. Y. Zhang, H. Wang, G. Z. Liang and A. J. Gu, Composites, Part B, 2015, 71, 96-102.

28 D. Shi, J. H. Yang, Z. H. Yao, Y. Wang, H. L. Huang, W. Jing, J. H. Yin and G. Costa, Polymer, 2001, 42, 5549-5557.

29 S. Mohanty, S. Nayak, S. Verma and S. Tripathy, J. Reinf. Plast. Compos., 2004, 23, 625-637.

30 R. Olley and D. Bassett, Polymer, 1982, 23, 1707-1710.

31 A. M. Díez-Pascual and M. Naffakh, ACS Appl. Mater. Interfaces, 2013, 5, 9691-9700.

32 O. Flores, A. Romo-Uribe, M. Romero-Guzmán, A. Gonzalez, R. Cruz-Silva and B. Campillo, J. Appl. Polym. Sci., 2009, 112, 934-941.

33 S. Garoushi, P. K. Vallittu and L. V. Lassila, Dent. Mater., 2007, 23, 1356-1362.

34 Y. W. Cao, J. C. Feng and P. Y. Wu, Polym. Eng. Sci., 2010, 50, 222-231.

35 G. P. Zhang, Z. Xin, J. Y. Yu, Q. D. Gui and S. Y. Wang, J. Macromol. Sci., Part B: Phys., 2003, 42, 467-478.

36 D. Libster, A. Aserin and N. Garti, Polym. Adv. Technol., 2007, 18, 685-695.

37 P. C. Vilanova, S. M. Ribas and G. M. Guzmán, Polymer, 1985, 26, 423-428.

38 F. Yang, T. Wu, M. Xiang and Y. Cao, Eur. Polym. J., 2017, 91, 134-148.

39 K. Cho, D. N. Saheb, H. C. Yang, B. I. Kang, J. Kim and S. S. Lee, Polymer, 2003, 44, 4053-4059.

40 C. Z. Geng, J. J. Su, C. Y. Zhou, H. W. Bai, G. H. Yang and Q. Fu, Compos. Sci. Technol., 2014, 96, 56-62.

41 J. J. Jia, X. Y. Sun, X. Y. Lin, X. Shen, Y. W. Mai and J. K. Kim, ACS Nano, 2014, 8, 5774-5783.

42 R. V. Silva, D. Spinelli, W. W. B. Filho, S. C. Neto, G. O. Chierice and J. R. Taroani, Compos. Sci. Technol., 2006, 66, 1328-1335. 